\title{
BRAF Rearrangement Analysis Was Performed
}

National Cancer Institute

\section{Source}

National Cancer Institute. BRAF Rearrangement Analysis Was Performed. NCI

Thesaurus. Code C160335.

An indication that BRAF rearrangement analysis was performed during the study. 